\title{
Monomeric CRP Aggravates Myocardial Injury After Myocardial Infarction by Polarizing the Macrophage to Pro-Inflammatory Phenotype Through JNK Signaling Pathway
}

\author{
Zhimin Zhal,* \\ Yujia Cheng ${ }^{2,3, *}$ \\ Lu Cao ${ }^{2}$ \\ Yanxia Qian ${ }^{2}$ \\ Xinjian $\mathrm{Liu}^{3,4}$ \\ Yan Guo' \\ Junhong Wang $\mathbb{D}^{2}$
}

'Department of Gerontology, The First Affiliated Hospital with Nanjing Medical University, Nanjing, People's Republic of China; ${ }^{2}$ Department of Cardiology, The First Affiliated Hospital with Nanjing Medical University, Nanjing, People's Republic of China; ${ }^{3}$ Key Laboratory of Antibody Techniques of National Health Commission, Nanjing Medical University, Nanjing, People's Republic of China; ${ }^{4}$ Department of Pathogen Biology, Nanjing Medical University, Nanjing, People's Republic of China

*These authors contributed equally to this work

\begin{abstract}
Objective: A polarized macrophage response plays a critical role in the pathophysiological process of myocardial infarction (MI). Several studies have shown a pro-inflammatory role for monomeric C-reactive protein (mCRP) in cardiovascular disease. However, the mechanism of how mCRP regulates macrophage phenotype switching remains unknown. In the present study, the effect of mCRP on macrophage polarization and its pathological function in myocardial repair after myocardial infarction was investigated.
\end{abstract}

Methods: MI was induced by permanent ligation of the left anterior descending coronary artery in ICR mice. Adult mice were injected with $\mathrm{mCRP}(2.5 \mathrm{mg} / \mathrm{kg})$ with or without SP600125 (15 mg/kg, JNK inhibitor) $45 \mathrm{~min}$ before MI. The cardiac function, scar size as well as cardiac fibrosis, infiltration of inflammatory cells, and the level of proteins in the JNK signaling pathway in infarcted myocardium were assessed. In addition, the phenotypic characterization of macrophages was further measured by ELISA, flow cytometry and quantitative RT-PCR in cultured THP-1 cells or peritoneal macrophages.

Results: Cardiac function deterioration, ventricular dilatation and fibrosis were exacerbated in mice pretreatment with $\mathrm{mCRP}$ following MI. Meanwhile, an increased accumulation of infiltrated inflammatory cells in infarcted myocardium was observed in the MCRP group. Moreover, activation of the JNK signaling pathway was markedly elevated in mCRP treated animals post-MI. In contrast, pharmacological inhibition of JNK phosphorylation activity by SP600125 muted the detrimental effects of mCRP in MI mice. Furthermore, in vitro and in vivo co-culture experiments showed that $\mathrm{mCRP}$ shifted macrophage polarization towards pro-inflammatory phenotypes, and this polarization could be abolished by sp600125.

Conclusion: Taken together, our results imply that mCRP impairs myocardial repair after myocardial infarction by polarizing the macrophages into the pro-inflammatory M1 phenotype via the JNK-dependent pathway.

Keywords: myocardial infarction, monomeric CRP, inflammation, macrophage polarization, JNK pathway

\section{Introduction}

Inflammation plays a pivotal role in cardiovascular disease such as atherosclerosis, acute myocardial infarction (MI) and heart failure. ${ }^{1-4}$ Recently, anti-inflammatory therapy had demonstrated valuable potentiality to alleviate the cardiac damage in acute myocardial infarction. ${ }^{5,6}$ Macrophages are considered to be the main participants in inflammation
Correspondence: Yan Guo; Junhong Wang Department of Gerontology, The First Univiated Hospital with Nanjing Medical District, Nanjing City, Jiangsu Province, 210009 , People's Republic of China Tel +86-25-68305083; +86-25-68308273 Email guoyan5I@hotmail.com; wangjunhong@jsph.org.cn 


\section{Graphical Abstract}

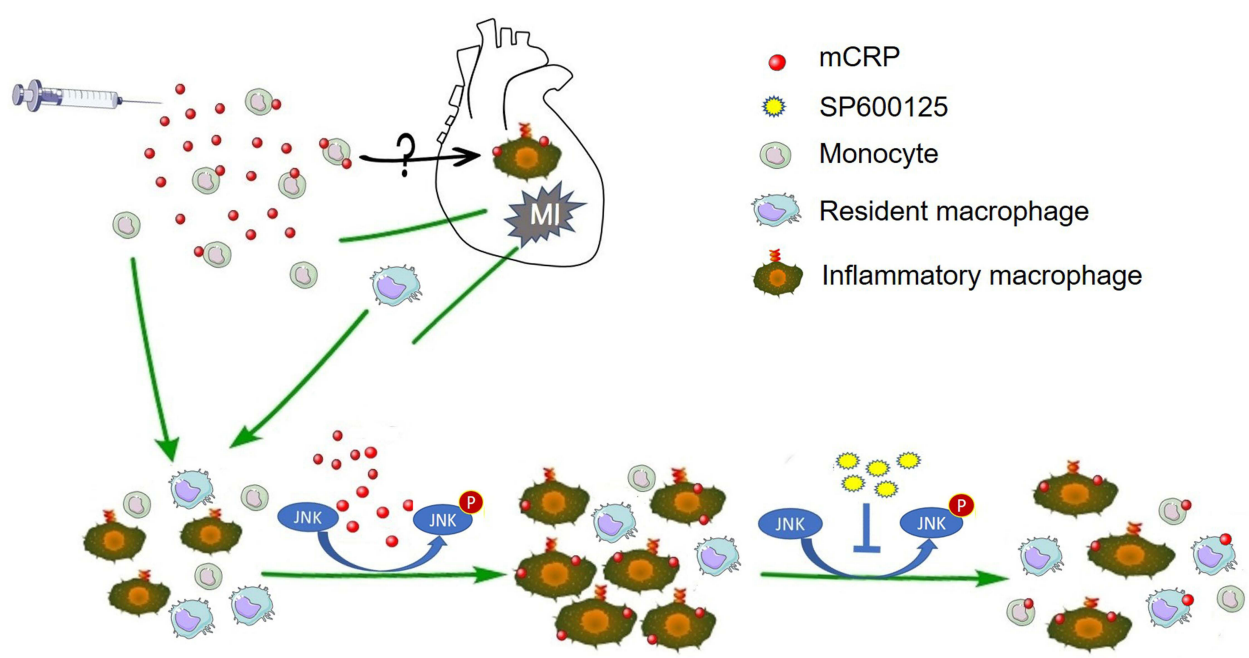

during the pathophysiological process of cardiovascular diseases. ${ }^{1,7,8}$ Meanwhile, they are highly plastic and assumed to function dependent on the micro-environmental cues. ${ }^{4,9,10}$ Classically, there are two functional subsets for macrophages: pro-inflammatory macrophages (M1) or anti-inflammatory /regulatory macrophages (M2). ${ }^{10,11} \mathrm{M} 1$ macrophages can produce pro-inflammatory cytokines such as TNF- $\alpha$, IL-6, IL-1 $\beta$, and reactive oxygen (ROS). In contrast, M2 macrophages highly express M2-associated anti-inflammatory cytokines, such as IL-10 and transforming growth factor- $\beta$ (TGF- $\beta$ ). M1 macrophages contribute to the pathogenesis of the inflammatory and degenerative diseases, they can also protect the host from infection. In contrast, M2 macrophages play an important role in suppressing the action of effector $\mathrm{T}$ cells to limit the amplification of inflammation, and, finally, are also beneficial for tissue repair. $^{12}$ Therefore, it is essential to coordinate the polarization of macrophage response in order to maintain the immunity and organism homeostasis. Understanding the molecular signals which drive macrophage polarization may give new clues to a variety of immunophysiological and pathological processes.

Monomeric CRP (mCRP) can be dissociated from its pentamer (pCRP) after binding to activated platelets, apoptotic/necrotic cells, or microparticles (MPs). ${ }^{13,14}$ However, it has distinct antigenicity-expressing neoepitopes differing from pCRP. This conformation rearrangement results in significant changes different from the biological activities of CRP. ${ }^{15,16}$ Furthermore, mCRP is reported to be involved in inflammatory events that may directly lead to atherosclerotic formation, ischemia/reperfusion injury, or acute myocardial infarction. $^{15,17,18}$ Monomeric CRP induces endothelial dysfunction, which also induces pro-inflammatory cytokine release from human monocytes. ${ }^{14,16}$ However, there are few data examining the direct effect and its possible signal pathways of mCRP on macrophage phenotype polarization and its pathophysiological functions after acute myocardial infarction. In the present study, we examined the hypothesis that mCRP may play a pro-inflammatory role on macrophage polarization and therefore impair the repair of the infarct myocardial after acute myocardial infarction.

\section{Materials and Methods}

\section{Reagents}

Recombinant human mCRP ( $>95 \%$ purity) with both cysteine residues mutated to alanine residues and with an added N-terminal formylmethionine residue was constructed and expressed in Escherichia coli as previously described and was decitraconylated by sequential dialysis. ${ }^{19}$ The mCRP preparation were characterized extensively by antigenicity detection with specific $\mathrm{mAb}$ and $1 / 20$ SDS-PAGE as we previously reported ${ }^{18}$ ensure its complete function and uniform structure showing no cross-contamination. Protein solutions were dialyzed to remove NaN3 and then passed through Detoxi-Gel columns (Pierce, Rockford, IL, USA) to remove endotoxin. The inhibitors, including CAPE (NF-kB inhibitor), and 
SP600126 (JNK inhibitor) were purchased from Selleck chem (Houston, TX, USA). The fluorescein-conjugated anti-mouse mAbs (F4/80-PE, CD11c-FITC and CD206APC) and their respective isotype controls were from eBiosience (San Diego, California, USA). Phorbolmyristate-acetate (PMA) and M- $\beta-C D$ were purchased from Sigma-Aldrich (St Louis, MO, USA).

\section{Cell Culture and Macrophages Generation}

The THP-1 cell lines were obtained from the American Type Culture Collection and grown in DMEM (Life Technologies) containing 10\% (v/v) heat-inactivated FBS Then cells were primed to M0 macrophages by PMA (100 $\mathrm{ng} / \mathrm{mL}$ ) for $72 \mathrm{~h}$ as previously described. ${ }^{20}$ Macrophages were exposed to serum free medium for $12 \mathrm{~h}$, and then incubated with mCRP to final concentration of $50 \mu \mathrm{g} / \mathrm{mL}$ for $24 \mathrm{~h}$. To further analyze whether JNK or NF- $\mathrm{KB}$ signals are involved in $\mathrm{mCRP}$-induced macrophage polarization, CAPE (a NF-kB inhibitor, $10 \mu \mathrm{M})^{21}$ or SP600125 (a JNK inhibitor, $10 \mu \mathrm{M})^{22}$ were cultured with macrophages for 24 $h$ to inhibit the activities of JNK or NF- $\kappa B$, respectively. In addition, $\mathrm{M}-\beta-\mathrm{CD}$ (lipid rafts disrupter, $5 \mathrm{mM})^{23}$ was added for $1 \mathrm{~h}$ before being co-cultured with $\mathrm{mCRP}$.

\section{RNA Isolation and Quantitative RT-PCR}

The total RNA was isolated using the TRIzol reagent (Invitrogen, US) in accordance with the manufacturer's instruction. The expression of target mRNA was quantitatively detected by two-step quantitative real-time PCR (Vazyme, Nanjing, China). The glyceraldehyde 3-phosphate dehydrogenase (GAPDH) was used as endogenous control, and the relative expression levels of target genes were determined by applying the DD cycle threshold method. All of the primers are listed in Supplementary Materials, Table 1.

\section{Mouse Peritoneal Macrophages Isolation}

All animal experiments were conducted in accordance with the National Institutes of Health Guide for the Care and Use of Laboratory Animals, and approved by the Animal Care and Use Committee of Nanjing Medical University (IACUC2003013). C57BL/6 female mice (6-8 weeks old, $\sim 20 \mathrm{~g}$ ) purchased from Cavens experimental animal co. Ltd (Changzhou, China) were injected intraperitoneally with sterile Brewer-thioglycollate medium ( $2 \mathrm{~mL}, 4 \% \mathrm{w} / \mathrm{v})$. At day 3 post-thioglycollate injection, the mice were randomly divided into different groups $(\mathrm{n}=4)$. A total volume of $0.2 \mathrm{~mL} \mathrm{mCRP}$ storage buffer (control), mCRP $(2.5 \mathrm{mg} / \mathrm{kg})$ and SP600125 $(15 \mathrm{mg} / \mathrm{kg})^{25}$ with/without mCRP was then injected i.p. for $24 \mathrm{~h}$, respectively (peritoneal). Before the mice were sacrificed, the peritoneal macrophages were harvested by peritoneal lavage with $10 \mathrm{~mL}$ sterile icecold PBS. Peritoneal fluid was collected and the levels of cytokines in peritoneal were then measured by ELISA.

\section{Myocardial Infarction Surgical Procedure}

ICR male mice were purchased from the production department of Nanjing Medical University (Nanjing, China). Mice were subjected to MI via occlusion of the left anterior descending coronary artery as previously described. ${ }^{24}$ In general, 8-week-old mice were anesthetized with $5 \%$ isoflurane and placed in a supine position on a heating pad, ventilated by a volume-controlled ventilator with $2.4 \%$ isoflurane. Then, the chest wall was shaved and a left thoracotomy was performed, a $6-0$ silk ligature was threaded through the left coronary artery for approximately $2-3 \mathrm{~mm}$ and myocardial ischemia was initiated by complete ligation of the left ascending (LAD) coronary artery. Mice with mere thoracotomy were treated as the sham group. SP600125 $(15 \mathrm{mg} / \mathrm{kg})$ and $\mathrm{mCRP}(2.5 \mathrm{mg} / \mathrm{kg})$ were given at $45 \mathrm{~min}$ before coronary artery ligation. Saline in equal volume was injected into the caudal vein as vehicle.

\section{Flow Cytometry}

Cells were washed in ice-cold flow cytometry buffer (2\% [v/v] FCS and 2 mM EDTA in PBS, pH 7.5), and then incubated with fluorescent labeled antibodies for $30 \mathrm{~min}$ and washed with buffer 3 times after blocking the unspecific binding. Appropriate isotype controls were used when necessary. After gated by forward and side scatter, F4/80+ cells were further chosen for analysis. Anti-mouse FITCCD11c, and APC-CD206 antibodies were then used to analysis the M1 macrophages from gated $\mathrm{F} 4 / 80+$ macrophages. ${ }^{25,26}$ Data were collected by a FACSCalibur flow cytometer (BD Biosciences) and analyzed with FlowJo software (Tree Star).

\section{Determination of Cytokine Levels}

The levels of TNF- $\alpha$ and IL-1 $\beta$ in the supernatants or peritoneal fluids were determined by ELISA (R\&D Systems, Minneapolis, MN) with the manufacturer's instructions. 


\section{Echocardiographic Examination}

Echocardiographic examination was conducted to evaluate the left ventricular systolic function In brief, on day 7 after occlusion, the heart tissues were viewed in the short-axis with M-mode VEVO 2100 system (Visual Sonics, Toronto, ON, Canada). Conventional measurements of the LV included left ventricular end-systolic diameter (LVESD), left ventricular end-diastolic diameter (LVEDD), ejection fraction (EF) and fractional shortening (FS). EF $(\%)=($ LVEDV-LVESV)/ LVESV $\times 100 \%$; FS $(\%)=($ LVEDD-LVESD $) /$ LVESD $\times 100 \%$.

\section{Triphenyltetrazolium Chloride (TTC) Staining}

The ratio of cardiac infarct size was assessed via TTC staining as previously described. ${ }^{27}$ The mouse heart was harvested at 7 $\mathrm{d}$ after MI. After being washed with saline, the heart tissues were frozen and cut into five slices, then stained in $1 \%$ TTC for $8-10 \mathrm{~min}$ and fixed in $4 \%(\mathrm{w} / \mathrm{v})$ paraformaldehyde for 30 min. The infarct area was characterized as a white region and size ratio was assessed using Image $\mathrm{J}$ software.

\section{Hematoxylin-Eosin (H\&E) Staining}

Mice were euthanized on either $7 \mathrm{~d}$ or $21 \mathrm{~d}$ after the surgical procedure. Cardiac tissue samples were dehydrated, embedded in paraffin and cut into 5 - $\mu \mathrm{m}$ thick sections. Paraffin-embedded sections were stained with hematoxylin for $5 \mathrm{~min}$ followed by $1 \%$ acid ethanol $(1 \% \mathrm{HCl}$ in $70 \%$ ethanol) and then rinsed in distilled water, stained with eosin for $3 \mathrm{~min}$ and re-immersed in alcohol and xylene. Finally, the sections were imaged under a light microscope and assessed for gross myocyte injury and the effects of interventions.

\section{Masson Staining}

Mice Hearts were harvested on $21 \mathrm{~d}$ after MI. The tissues were simply trimmed to dissociate the upper part of ligation and immersed in $4 \%$ paraformaldehyde for $48 \mathrm{~h}$ at $4{ }^{\circ} \mathrm{C}$. After dehydration through alcohol gradient, the samples were embedded in paraffin wax and cut into $5 \mathrm{~mm}$ thick sections. Slices then were stained for collagen fibers with Masson's Trichrome staining. In Masson-stained sections, myocardial cells were stained red while collagen was stained blue. The photographs were taken with a light microscope.

\section{Western Blotting Analysis}

Western blotting analysis was performed as previously described $^{28}$ using anti-p-JNK (Thr183/Tyr185,4668S, CST, America), anti-JNK (66210-1-Ig, Proteintech, America),
anti-ASK1 (3762S, CST, America), and anti-Smad2/3 (8685S, CST, America) antibodies. Mouse monoclonal antiGAPDH antibody (Cat No. 60004-1-Ig, Proteintech, America) was used as the internal control.

\section{Statistical Analysis}

All data are presented as independent mean \pm standard deviation unless otherwise indicated. Results were analyzed with unpaired $t$-test within 2 groups if both groups were normally distributed. The statistical significance of the differences between 3 or more groups was analyzed through the one-way analysis of variance (ANOVA), followed by multiple comparison test. A $p$ value $<0.05$ was considered to be statistically significant. All of the calculations were performed using the SPSS 20.0 for Windows (SPSS Inc, Illinois, USA) and GraphPad Prism 8 software.

\section{Results \\ mCRP Primed Macrophages to Pro-Inflammatory Phenotype}

After incubation for 24 hours, mCRP resulted in significantly increased pro-inflammatory macrophage associated gene expression of IL-1 $\beta$, TNF- $\alpha$, CD40 and CD80 (Figure 1A, B and Supplementary Figure 1) as well as in protein level measured by ELISA (Figure 2A and B). Peritoneal treatment with mCRP further primed a significant pro-inflammatory M1 phenotypic macrophage when measured by flow cytometry (Figure $3 \mathrm{~A}-\mathrm{C}$ ). Likewise, administrating $\mathrm{mCRP}$ further significantly increased the level of IL-1 $\beta$ and TNF- $\alpha$ in cultured supernatants (Figure 4A and B). Interestingly, the antiinflammatory macrophage associated genes such as CD206 and CD200R did not show any substantial changes when incubated with mCRP (Supplementary Figure 2). Taken together, these data might indicate that mCRP plays a role in determining macrophage skewing to proinflammatory phenotype.

\section{mCRP Primed Macrophages to MI Phenotype via Activation of the JNK Pathway}

We then explored the possible mechanism underlying macrophage polarization by mCRP. Macrophages were exposed to serum free medium for $12 \mathrm{~h}$, and then incubated with mCRP with or without the inhibitors, respectively, for $24 \mathrm{~h}$. Because NF- $\mathrm{BB}$ signaling pathway was reported to involve in pCRP induced macrophage 
A

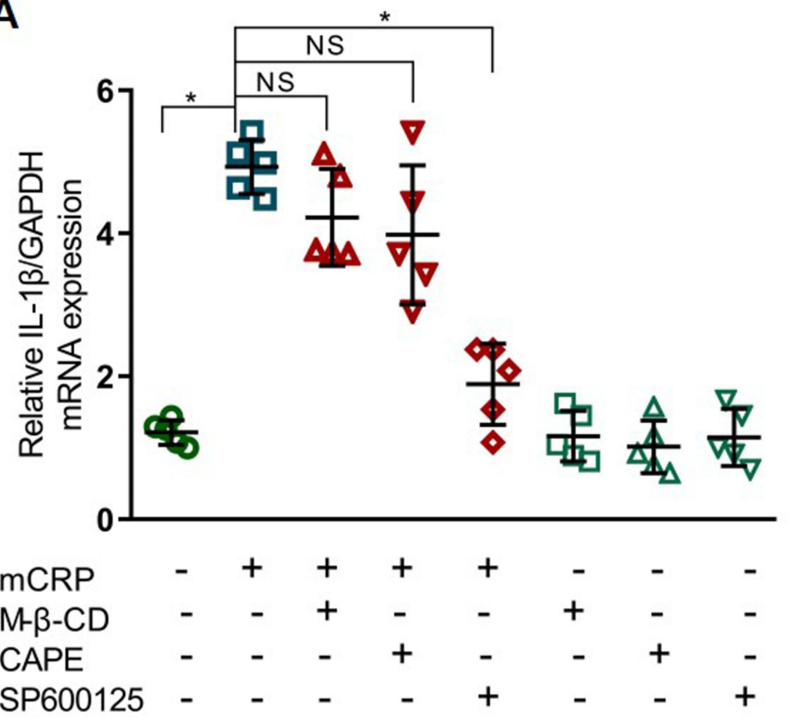

B

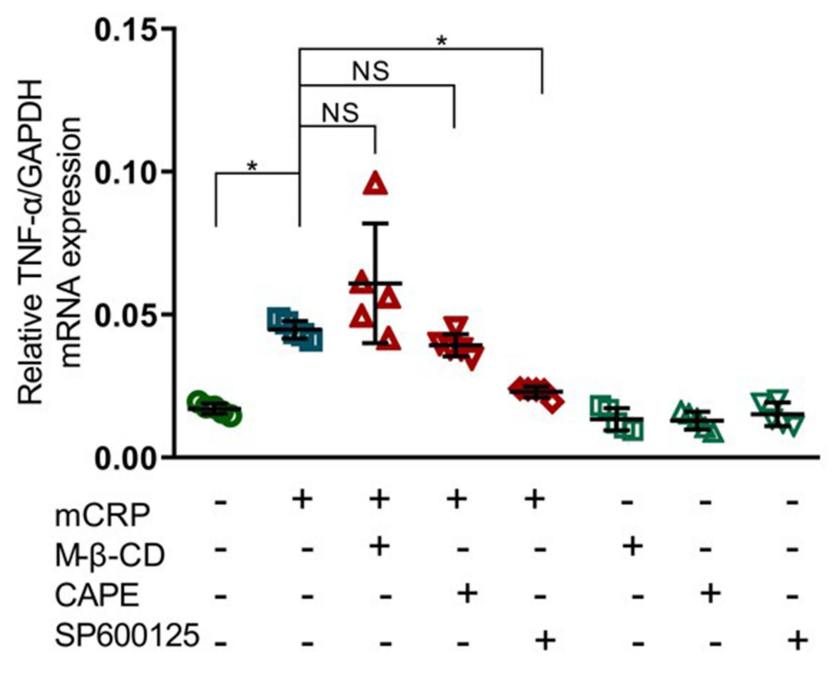

Figure I Effects of $m C R P$ and the inhibitors in the expression of (A) IL-I $\beta$ and (B) TNF- $\alpha$ in cultured macrophages. The mRNA expression of IL-I $\beta$ and TNF- $\alpha$ in the THPI cells incubated with $\mathrm{mCRP}(50 \mu \mathrm{g} / \mathrm{mL})$ for $24 \mathrm{~h}$ was blocked by the JNK inhibitor (SP600 I25, $10 \mu \mathrm{M})$ instead of the NF-kB inhibitor (CAPE, $10 \mu \mathrm{M})$ and lipid rafts disrupter $(\mathrm{M}-\beta-\mathrm{CD}, 5 \mathrm{mM})$. Data are represented as mean $\pm \mathrm{SD}$, $* p<0.05$.

Abbreviation: NS, not significant.

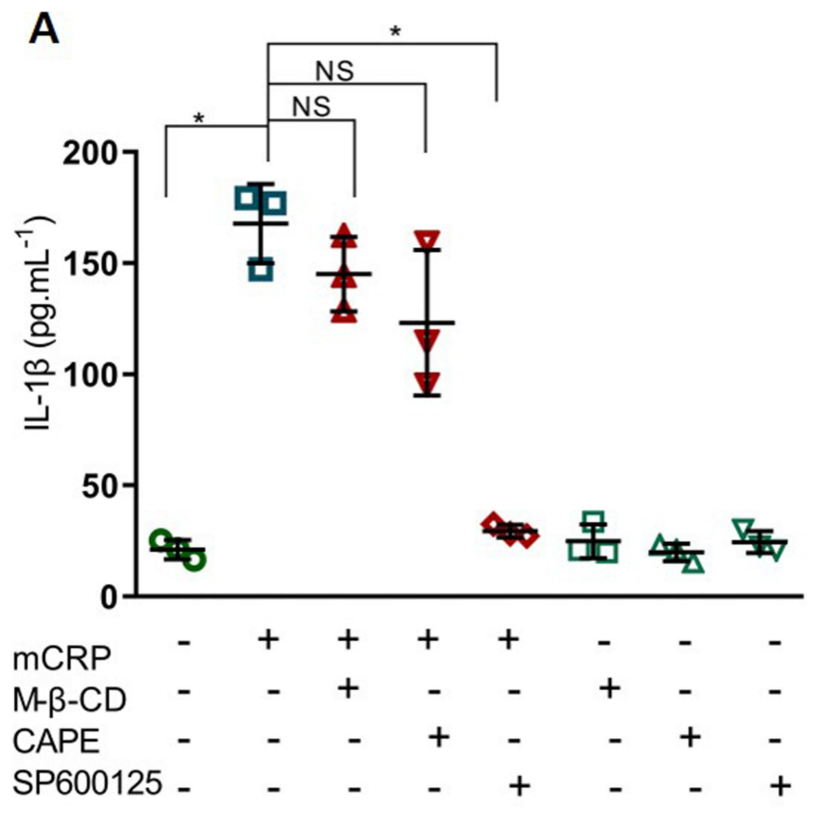

B

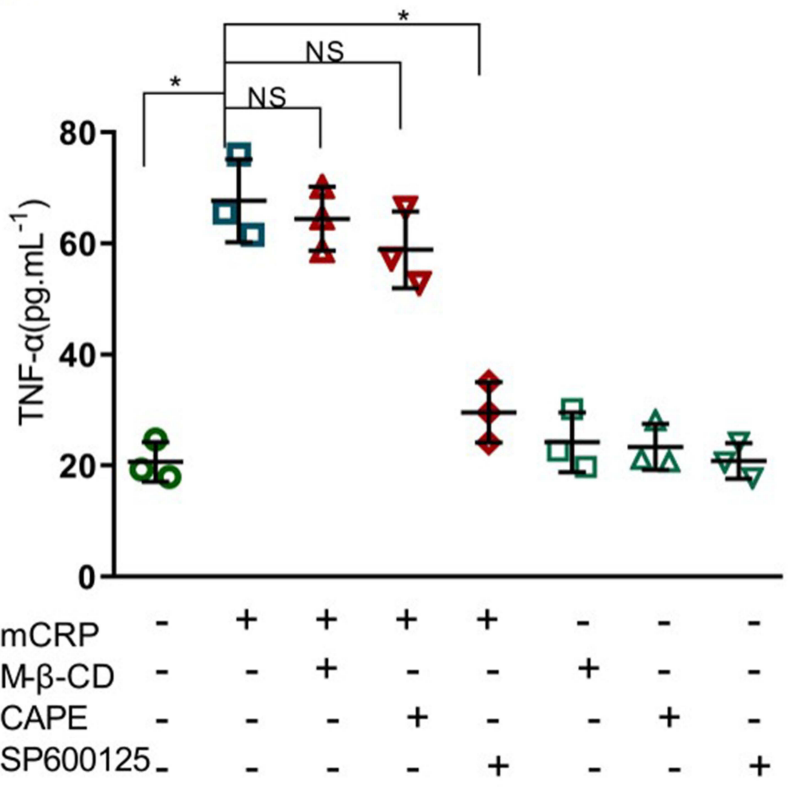

Figure 2 Effects of $m C R P$ and the inhibitors on the protein levels of (A) IL-I $\beta$ and (B) TNF- $\alpha$ in cultured macrophages measured by ELISA. The protein levels of IL-I $\beta$ and TNF- $\alpha$ in the supernatant of cultured THP-I cells incubated with $\mathrm{mCRP}(50 \mu \mathrm{g} / \mathrm{mL})$ for $24 \mathrm{~h}$ was blocked by the JNK inhibitor (SP600I25, $10 \mu \mathrm{M})$ instead of the NF-KB inhibitor (CAPE, $10 \mu \mathrm{M})$ and lipid rafts disrupter $(M-\beta-C D, 5 \mathrm{mM})$. Data are represented as mean $\pm \mathrm{SD},{ }^{*} p<0.05$.

Abbreviation: NS, not significant.

polarization, CAPE was used to inhibit the activation of $\mathrm{NF}-\mathrm{\kappa B}$ to see if they share the same signaling pathway. However, the levels of M1 signature biomarkers of M1 macrophages did not change significantly in mCRP treated macrophages when CAPE was administrated to the cells.
In contrast, the JNK inhibitor, SP600125 markedly inhibited the production of $\mathrm{M} 1$ associated biomarkers including TNF- $\alpha$ and IL- $1 \beta$ both in mRNA and protein levels induced by mCRP in cultured cells. (Figure 1A, B and Supplementary Figure 3). 
A

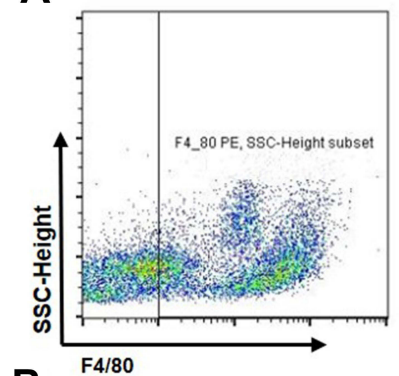

B $\quad \mathrm{F} 4 / 80 \mathrm{mCRP} 2 \mathrm{mg} / \mathrm{kg}$

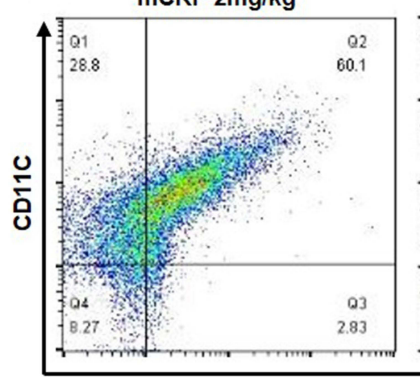

B

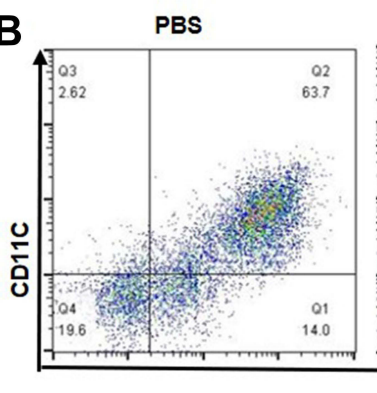

$\mathrm{mCRP} 0.8 \mathrm{mg} / \mathrm{kg}$
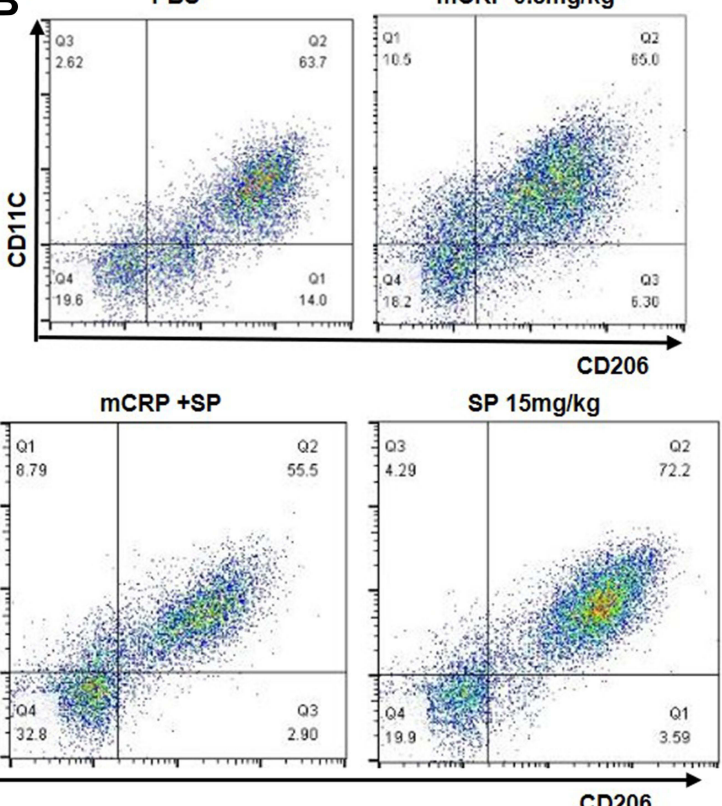

C

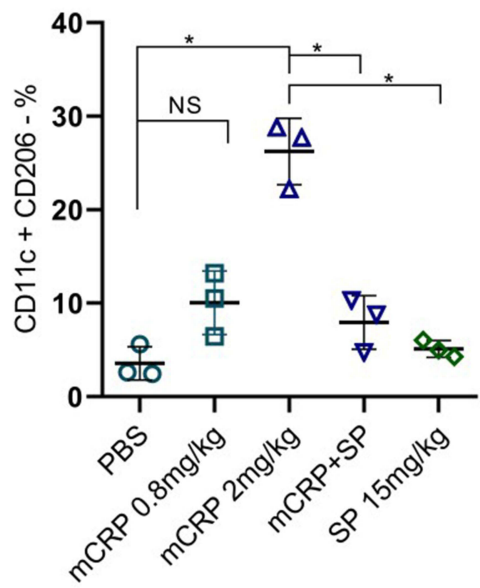

Figure 3 Monomeric CRP polarized the mouse peritoneal macrophages to pro-inflammatory phenotypes in C57BL/6 mice in vivo. Peritoneal macrophages were collected and F4/80, CDI Ic, and CD206 expression were analyzed by flow cytometry at $24 \mathrm{~h}$ after intraperitoneal injection. (A) All cells are gated by F4/80 staining, and (B) then the subtypes were differentiated by CDI Ic and CD206 staining in each group as indicated respectively. (C) Data represented the mean \pm SD of five groups. $n=3$ per group. $* p<0.05$.

Abbreviation: NS, not significant.

A

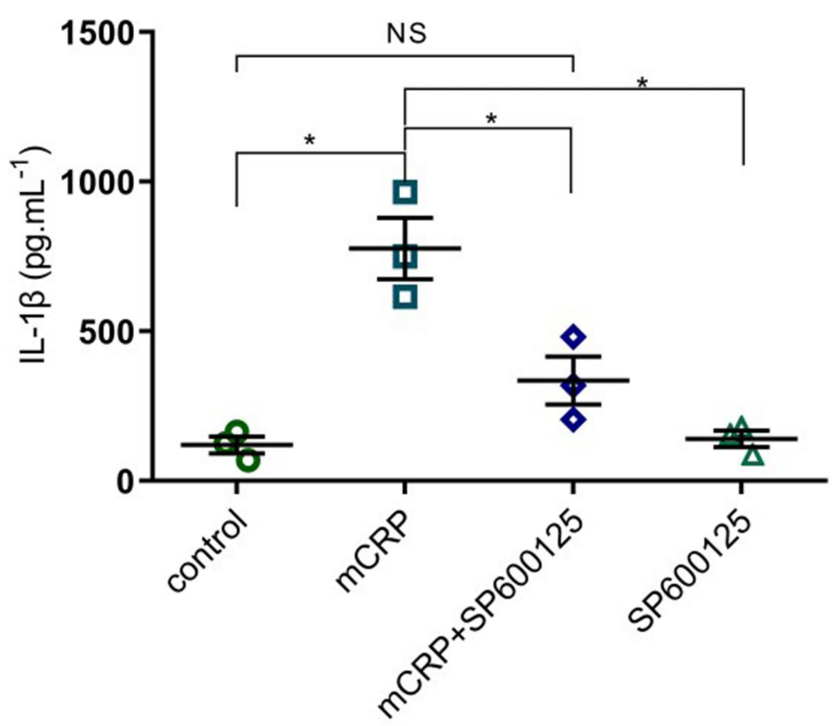

B

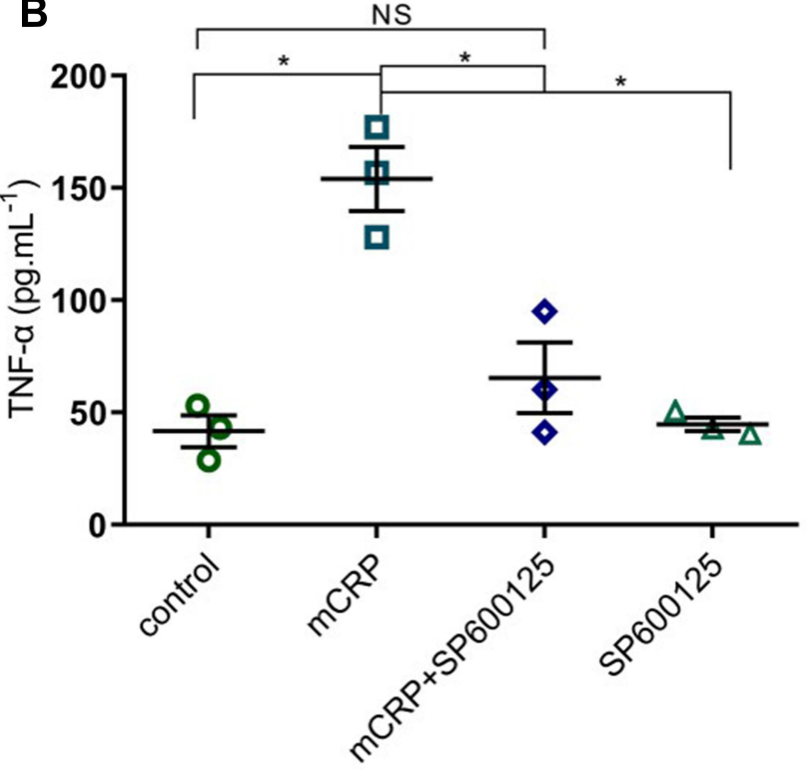

Figure 4 Effects of $m C R P$ and the JNK inhibitor, SP600I 25 on the protein levels of (A) IL-I $\beta$ and (B) TNF- $\alpha$ in mice peritoneal fluids measured by ELISA. The protein levels of IL-I $\beta$ and TNF- $\alpha$ in the peritoneal fluid stimulated by mCRP $(2.5 \mathrm{mg} / \mathrm{kg}$ ) for $24 \mathrm{~h}$ was blocked by the JNK inhibitor (SP600I25, $10 \mu \mathrm{M})$. $\mathrm{n}=3$ per group. Data are represented as mean $\pm \mathrm{SD}, * p<0.05$.

Abbreviation: NS, not significant.

To further analyze whether JNK signals are involved in mCRP-induced macrophage polarization, SP600125 was used to inhibit the activation of JNK in vivo. As shown in Figure 3, peritoneal treatment with SP600125 caused a substantial decrease of peritoneal pro-inflammatory macrophages when compared to the mCRP group. To 
gain insights into the mechanisms involved in mCRPinduced JNK activation in macrophages, we examined the expression of JNK, its phosphorylation form p-JNK and signaling molecules downstream and upstream of JNK. Of note, the expression of phosphorylated JNK, as detected by phospho-specific antibody recognition of the Thr183/Tyr185 site of JNK (p-JNK Thy183/Tyr 185) was substantially increased upon the stimulation of mCRP both in vivo and in vitro, which was downregulated by the JNK inhibitor, SP600125 (Figure 5A, B, D and E). Therefore, we revealed a selectively enhanced activation of JNK pathway caused by mCRP, which was most likely responsible for the skewing of proinflammatory macrophage development. Since ASK1 is the most common upstream molecular of JNK pathway, we subsequently detected the expression of ASK1 after mCRP incubation in macrophages. However, the protein level of ASK1 did not change with mCRP stimulation, indicating that ASK1 may not be involved in mCRP-induced macrophage polarization (Figure 5A and C).

Furthermore, as lipid rafts were reported to play an important role in mCRP-induced pathological functions in platelet and endothelial cells, we then evaluated the role of lipid rafts in mCRP induced macrophage polarization by incubating the cells with the lipid rafts disrupter, $\mathrm{M}-\beta-\mathrm{CD}$. However, even $5 \mathrm{mM}$ of $\mathrm{M}-\beta-\mathrm{CD}$ caused no significant changes in the production of IL- $1 \beta$ and TNF- $\alpha$ induced by mCRP (Figures 1 and 2).

\section{mCRP Impairs Myocardial Repair After Acute Myocardial Infarction by Polarizing Macrophage to Pro-Inflammatory Phenotype}

Macrophage polarization is a common event after MI, whereas macrophage phenotypes control the cardiac
A

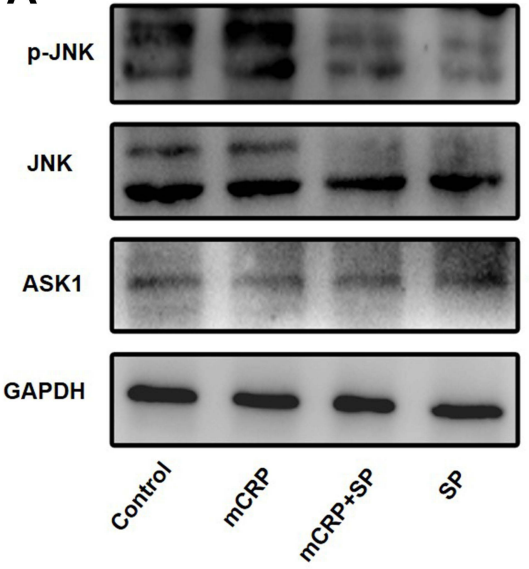

D

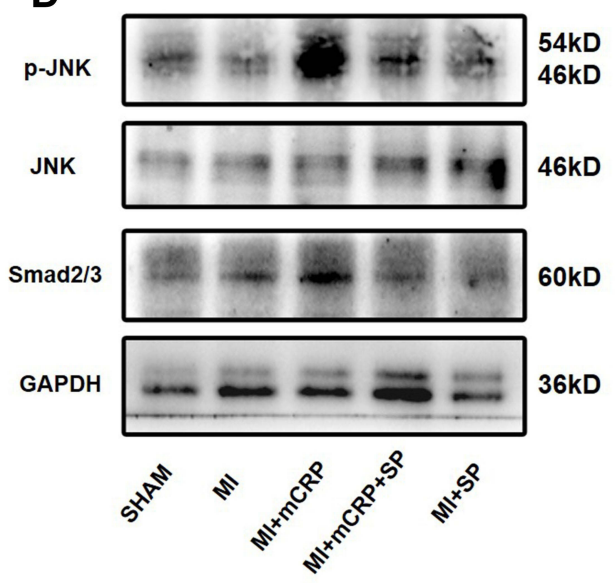

B $54 \mathrm{kD}$ 46kD

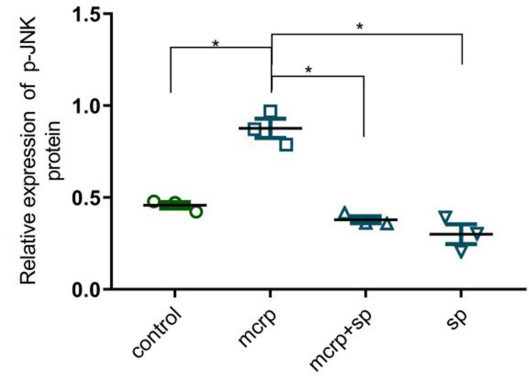

E

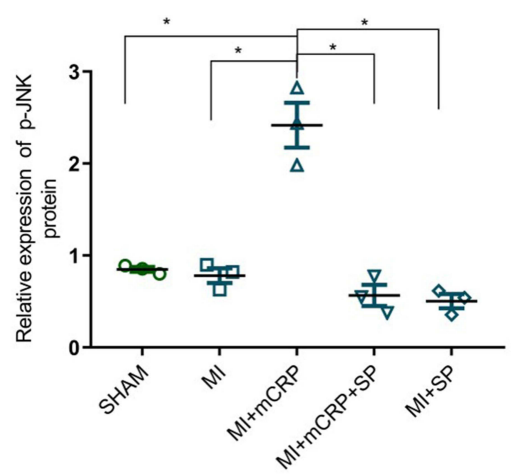

C

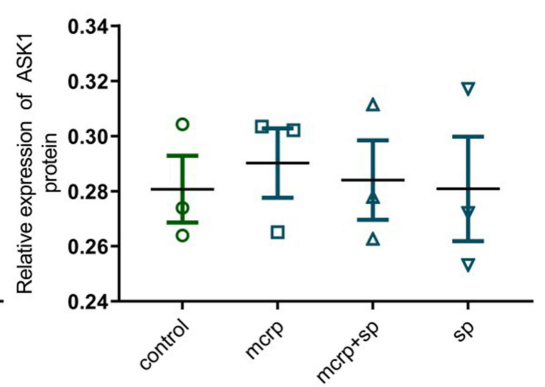

$\mathbf{F}$

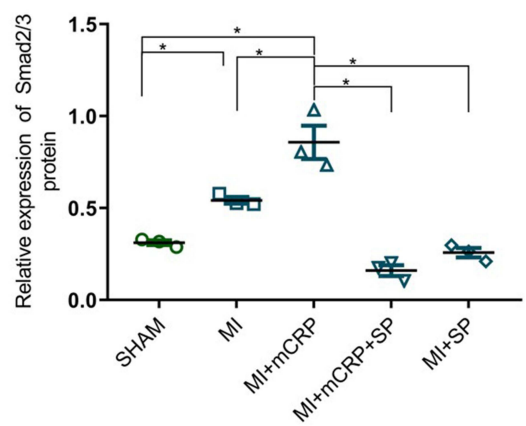

Figure 5 Effects of $\mathrm{mCRP}$ and the JNK inhibitor, SP600I25 on the protein levels of JNK signaling pathway. (A) Representative images of P-JNK, JNK, and ASKI in THP-I cells incubated with $\mathrm{mCRP}(50 \mu \mathrm{g} / \mathrm{mL})$ and/or JNK inhibitor (SP600I25, $10 \mu \mathrm{M})$ for $8 \mathrm{~h}$. (B and C) Quantitative analysis of P-JNK, and ASKI in cultured THP-I cells. (D) Representative images of $\mathrm{p}-\mathrm{JNK}$, JNK, and Smad2/3 in infarcted myocardium 7 days after myocardial infarction. (E and $\mathbf{F}$ ) Quantitative analysis of $\mathrm{p}-\mathrm{JNK}$, and Smad2/3 in the infarcted area in mice. $n=3$ per group. Data are represented as mean $\pm S D, * p<0.05$. 
function recovery and the degree of cardiac damage and repair. ${ }^{29} \mathrm{We}$, therefore, investigated whether $\mathrm{mCRP}$ affects macrophage polarization and its impact on cardiac function in vivo after MI. We initially examined the cardiac function in mCRP treated and control mice at baseline and 1-week post MI using echocardiographic analysis. Compared to the MI control, LVEF in mCRP-treated mice was substantial reduced from an average of $58.2 \%$ to $45.49 \%$ ( $12.7 \%$ reduction compared to baseline) postMI. In contrast, we observed increased LVEF in mice when JNK inhibitor, SP600125 was simultaneously administrated with mCRP, from $45.49 \%$ in mCRP group to $60.52 \%$ post-MI (Figure $6 \mathrm{~A}$ and B). Consistently, The FS of each group showed similar changes to LVEF (Figure 6C). Likewise, TTC staining further revealed that significant larger infarcted area in mCRP group than in MI group ( $34.47 \%$ vs $23.28 \% p<0.05)$, and those detrimental effects of mCRP could be reversed by JNK inhibitor, SP600125 (Figure 6D and E).

Next, we investigated whether JNK signaling pathway is involved in the impairment of cardiac function in mCRP mice after MI and whether its impairment is due to changes in macrophage subpopulation. At day 7 post-MI, a significantly increased phosphorylated level of JNK (Thr183/Tyr185) was observed in the infarcted area of mice administrated with mCRP. Likewise, SP600125 inhibited the level of p-JNK increased by mCRP in the infarcted myocardium (Figure 5D and E). H\&E examination further demonstrated that significantly more numbers of infiltrated inflammatory cells appeared in the infarcted myocardium of mice administrated with mCRP in comparison to the mice only with MI procedure at day 7 post-MI. Surprisingly, more infiltrated inflammatory cells remained in the infarcted myocardium in the MCRP group at day 21 post-MI, indicating the prolonged inflammatory duration caused by mCRP after MI (Figure 7A). However, when pretreated with SP600125, not only the infiltrated inflammatory cells but also the number of pro-inflammatory macrophages in infarcted myocardium decreased significantly compared with the mCRP group (Figure 7A). Consequently, Masson trichromatic staining at 21-days after MI showed more collagen fiber deposition in the infarcted area in the mCRP group compared with the control MI group, and SP600125 further reduced mCRPinduced collagen deposition in infarcted myocardium (Figure 7B). Interestingly, the level of $\operatorname{smad} 2 / 3$ increased significantly in the infarcted myocardium 7-days post-MI in the mCRP group, further indicating the over-activation of inflammation by mCRP after myocardial infarction.
However, the expression of smad2/3 decreased significantly when the mice were pre-administered with sp600125 $30 \mathrm{~min}$ before the myocardial infarction (Figure 5D and F).

\section{Discussion}

Macrophages are extremely heterogeneous displaying both pro- and anti-inflammatory functions. Specific stimuli from both endogenous and exogenous environments are essential for functional phenotypic macrophages. ${ }^{30}$ Previous studies indicated that pro-inflammatory to anti-inflammatory phenotypic shift in the macrophage population plays a key role in the process of cardiac repair after an acute myocardial infarction (AMI) ${ }^{8,18,31}$ Moreover, some molecules are reported to have the ability to prime the macrophages from M1 to M2. ${ }^{31}$ Therefore, understanding the potential molecules and the underlying mechanisms of this macrophage phenotypic plasticity may be beneficial for the treatment of AMI.

In the present study, we investigated the effects of mCRP on macrophage phenotypes, its underlying molecular mechanisms and its consequent pathological function in the repairing of myocardium after MI. Our results highlighted that $\mathrm{mCRP}$ promotes pro-inflammatory macrophage development in cultured macrophages. We further analyzed the phenotype of peritoneal macrophage in vivo. We found that mCRP significantly upregulated the number of pro-inflammatory macrophages and the expression of pro-inflammatory profiling biomarkers such as TNF- $\alpha$ and IL- $1 \beta$ in the peritoneal fluid. The importance of this finding is highlighted by the findings that mCRP was involved in the pathological process after acute myocardial infarction. ${ }^{14,31} \mathrm{We}$ and other studies further illustrated that increased localization of mCRP was observed in ischemic stroke or the infarcted myocardium after myocardial infarction. ${ }^{18,32,33}$ Here, we further demonstrated that mCRP increased and prolonged the duration of proinflammatory cells in infarcted myocardium after MI through the JNK pathway. In addition, this accentuation and prolongation of the post-infarction inflammatory reaction induced by mCRP impairs the reparative response after acute myocardial infarction, which was evident by significantly decreased left ventricular systolic function as well as increased damage to the myocardium at 7 or 21 days after myocardial infarction. As increased and prolonged pro-inflammatory macrophage residual in infarcted myocardium was reported to closely relate to the adverse clinical outcomes, ${ }^{34}$ our results indicated that mCRP delayed the process of the cardiac repair after MI, which 
A

SHAM
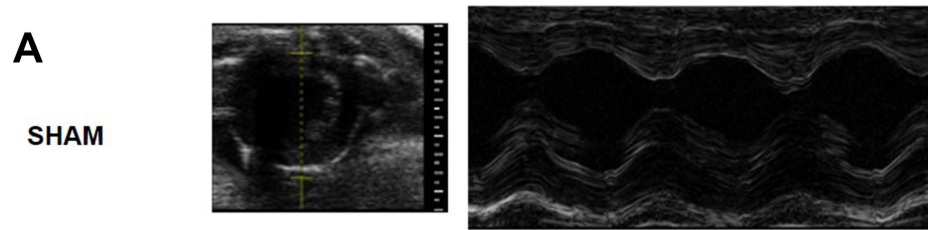

MI
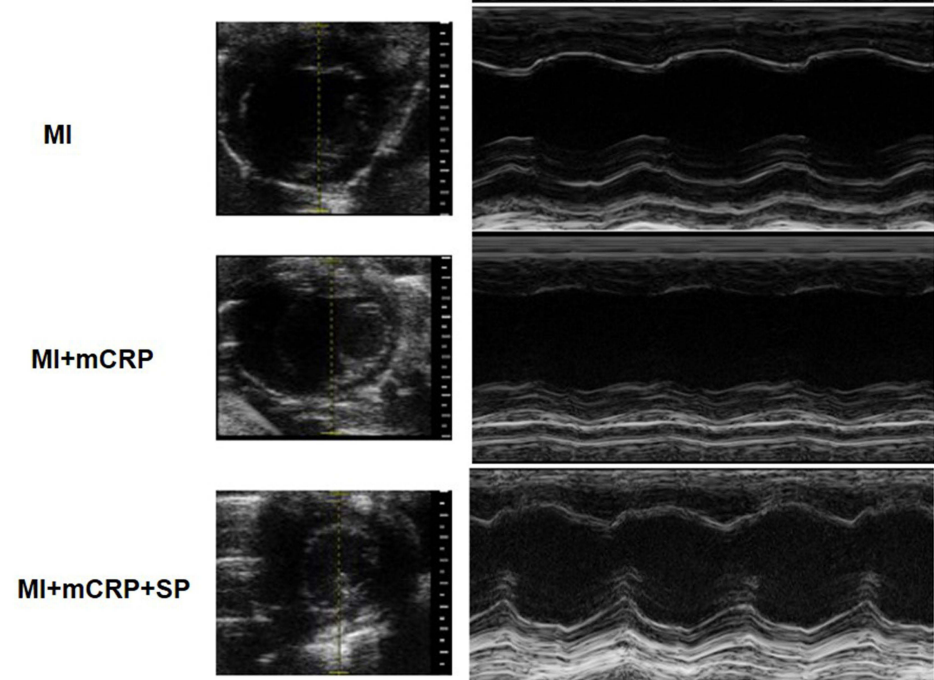

Ml+mCRP+SP

MI+SP
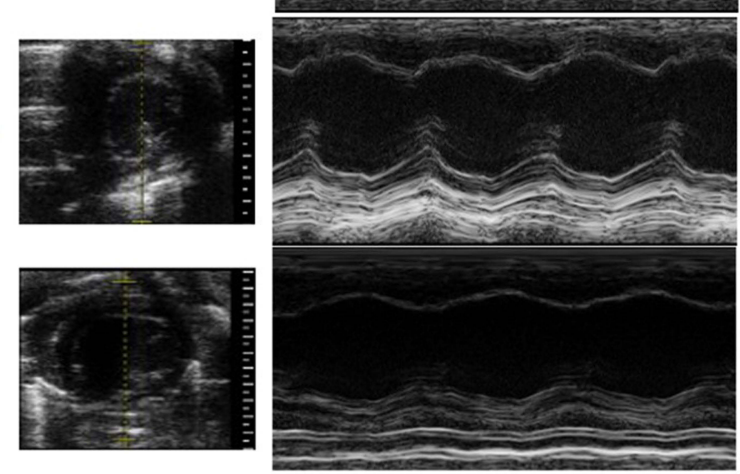

D SHAM

MI

MI
MI+ mCRP

MI+ SP

$M I+m C R P+S P$
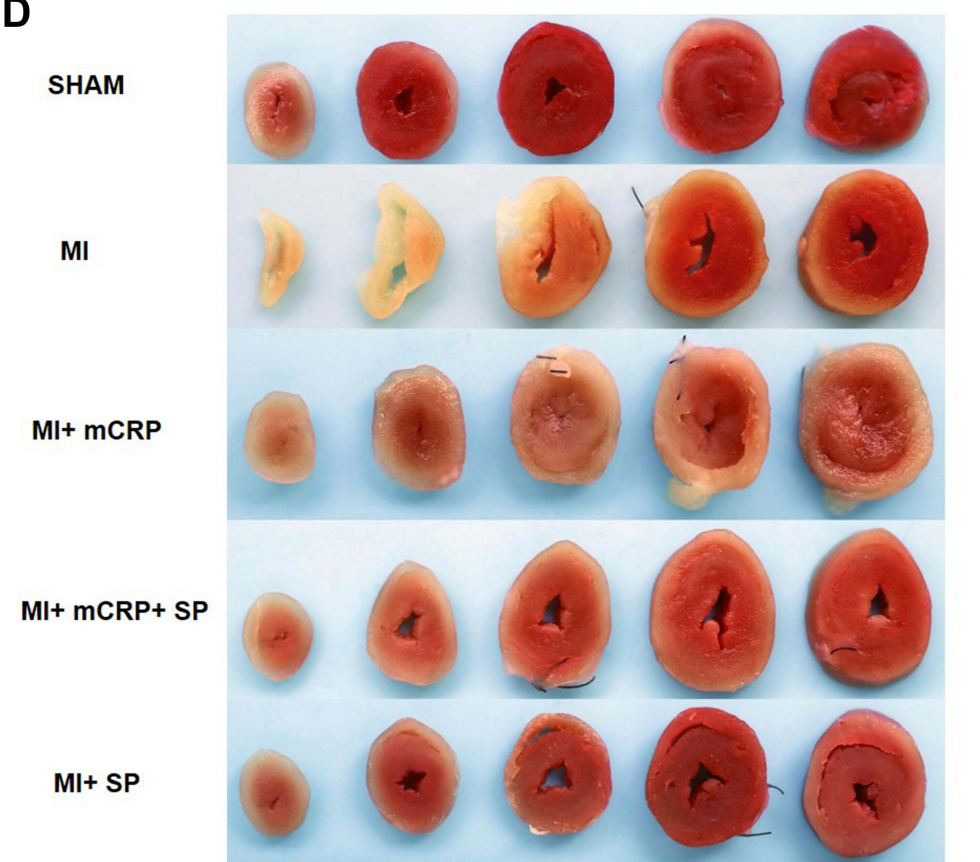

B

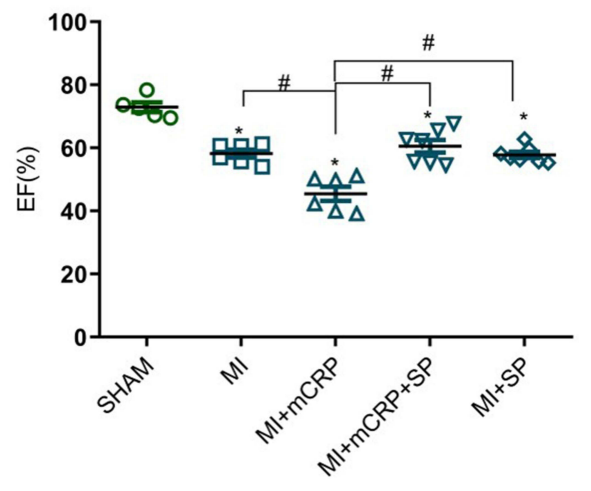

C

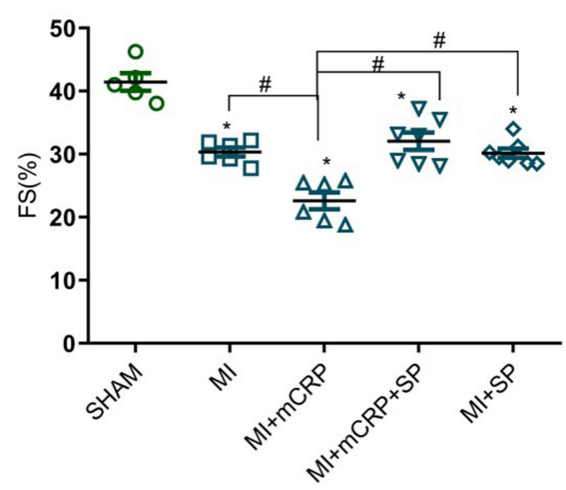

E

Day 7 post-MI

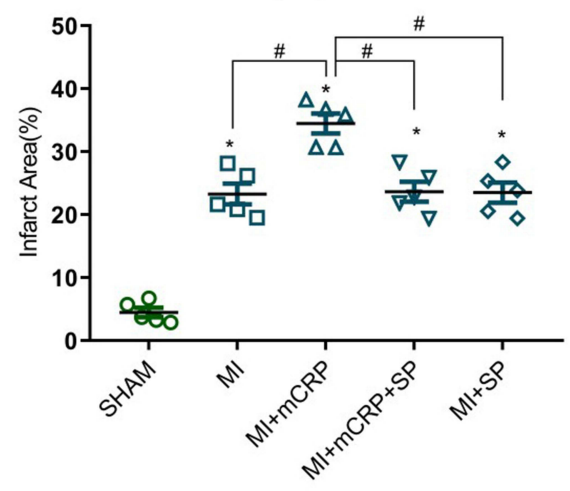

Figure 6 Effects of $\mathrm{mCRP}$ and the JNK inhibitor (SP600I25) on the cardiac function and infarct size in MI mice. (A) Representative long-axis or short-axis echocardiographic images of mice at 7-days after myocardial infarction in each group. (B and C) Quantitative analysis of cardiac function of EF (\%) and FS (\%) in mice in each group. (D) Representative TTC stained image of each mice group. The area at risk is stained as red and the infarcted myocardium is stained as white. (E) Quantitative analysis of ratio of infarct size. $n=5-7$ per group. Data are presented as mean \pm SD. ${ }^{p} p<0.05$ vs sham group; ${ }^{*} p<0.05$. 


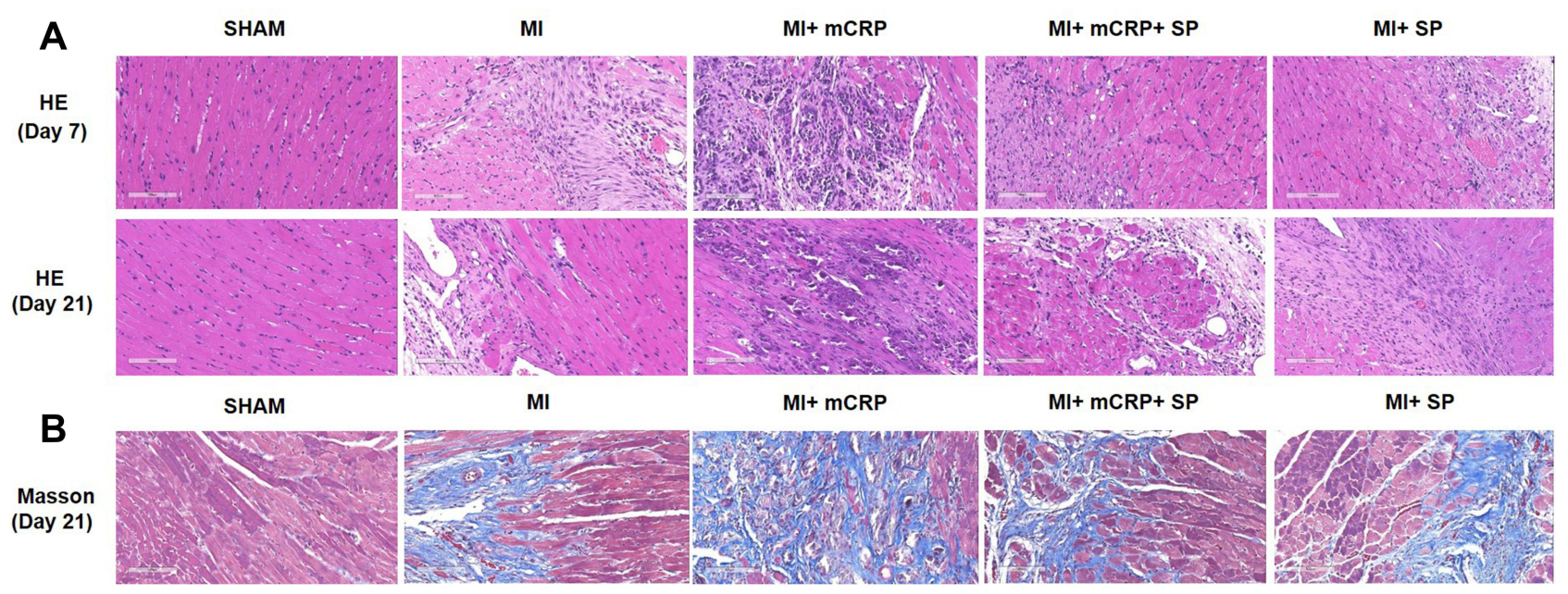

Figure 7 Effects of mCRP and the JNK inhibitor (SP600I25) on histopathological changes in mice. (A) Representative images of HE staining of infarction area (200× magnification) at 7d and $2 \mathrm{Id}$ post-MI in mice in each group. Scale bars $100 \mu \mathrm{m}$. (B) Representative images of Masson trichrome staining of infarction tissue in each group (200× magnification) at $2 \mathrm{I} d$ post-MI in mice. Scale bars $100 \mu \mathrm{m}$.

may become a prognostic factor for the clinical outcome of patients after myocardial infarction in the future.

As macrophage polarization involves the activation of several transcription factors, the possible pathways in mCRP-polarized M1 macrophages were then investigated in this study. The JNK pathway plays an important role in regulating the inflammatory cytokines expression through phosphorylation of transcription factors. ${ }^{35}$ In addition, macrophage-specific JNK-deficiency was reported to cause reduced M1 differentiation. ${ }^{36}$ To investigate whether the JNK pathway is involved in mCRP-induced macrophage polarization, JNK specific inhibitor, SP600125 was used to study the role of a JNK signaling pathway in macrophage subpopulation shifting after mCRP stimulation. Our study demonstrated that mCRP activates JNK signaling pathway by phosphorylation at Thr183/Tyr 185 sites, which subsequently polarizing macrophages to the pro-inflammatory sub-phenotype both in vitro and in vivo. When inhibited by the specific JNK inhibitor, a significantly decreased proportion of pro-inflammatory macrophage and the production of M1-related cytokines as well as downregulated phosphorylated JNK was observed both in vitro and in vivo. Importantly, the deleterious effects of mCRP, which augments inflammation and hinders subsequent myocardial repair after myocardial infarction, were partially ameliorated by the JNK inhibitor, suggesting the role of the JNK pathway in the pathogenesis of mCRPinduced cardiac remodeling. The JNK pathway was reported to be essential for M1 macrophage polarization in HFD-fed mice, and lack of JNK decreases the expression of
M1 phenotypic related genes and increased the M2 related genes in adipose tissue macrophages. ${ }^{37}$ Furthermore, NF$\kappa B$ pathway was also reported to be associated with pCRPinduced M1 macrophage polarization, ${ }^{38}$ however, inhibition of NF- $\mathrm{kB}$ activation by its inhibitor had no effect on the production of mCRP-induced M1-related cytokines in our study. Therefore, our results may suggest that although $\mathrm{mCRP}$ was the monomeric form of CRP, they shared different signaling pathways in showing the M1 macrophage polarization bias. To sum up, our results demonstrated that JNK activation is required for mCRP-induced M1 macrophage polarization.

It is still unclear how macrophages recognize mCRP and dispense signals to its downstream signal molecules. Therefore, we investigated the interaction of mCRP and cell membranes. The lipid rafts were observed to be the docking site for localizing and sensing mCRP's signals to downstream targets in platelets and endothelial cells. However, the lipid rafts disrupter M- $\beta-C D$ was not able to block the mCRP-induced M1 macrophage polarization in our study, indicating that the membrane docking site, other than lipid rafts, may be involved in the mCRP induced macrophage polarization.

\section{Limitations}

The limitation of this study is that we only focus on studying the role of pro-inflammatory M1 phenotype macrophages in myocardial repair after myocardial infarction. However, this definition may be an oversimplified way to classify the phenotype of macrophages due to the 
phenotypic change of macrophages is a dynamic continuous process. In addition, other inflammatory cells such as neutrophils may also participate in the process of myocardial inflammation and injury after myocardial infarction caused by mCRP. Further studies such as macrophage depletion experiments can further clarify the role of macrophage on the process of mCRP caused myocardial injury after myocardial infarction.

\section{Conclusion}

In conclusion, our data revealed a relationship between mCRP and M1 macrophage polarization which is essential for mediating accentuation and prolongation of the postinfarction inflammatory reaction after myocardial infarction. As the specific stimuli are pivots for priming the phenotype of the macrophage population, mCRP might be a useful therapeutic target for the treatment of myocardial infarction by blocking the mCRP effects and decreasing the pro-inflammatory macrophage population. Hence, it would be interesting to undergo further studies to investigate the role of mCRP in the treatment of acute myocardial infarction.

\section{Highlights}

- mCRP aggravates myocardial injury after myocardial infarction.

- mCRP polarizes macrophage to a pro-inflammatory phenotype.

- mCRP primes macrophage to a pro-inflammatory phenotype through the JNK pathway.

\section{Funding}

This work was supported by the National Natural Science Foundation of China (NSFC 82170269, 81570328, Wang JH), the Jiangsu Province's Key Provincial Talents Program (ZDRCB2016005), Jiangsu Students' platform for innovation and entrepreneurship training program (2020103112008Y) and the Foundation of Clinical Medical Research Center of Yili Autonomous Prefecture (YL2020ms09, Wang JH)

\section{Disclosure}

The authors report no conflicts of interest in this work.

\section{References}

1. Prabhu SD, Frangogiannis NG. The biological basis for cardiac repair after myocardial infarction: from inflammation to fibrosis. Circ Res. 2016;119(1):91-112. doi:10.1161/CIRCRESAHA.116.303577
2. Heidenreich P. Inflammation and heart failure: therapeutic or diagnostic opportunity? J Am Coll Cardiol. 2017;69(10):1286-1287. doi:10.1016/j.jacc.2017.01.013

3. Hansson GK. Inflammation and atherosclerosis: the end of a controversy. Circulation. 2017;136(20):1875-1877. doi:10.1161/ CIRCULATIONAHA.117.030484

4. Ong SB, Hernandez-Resendiz S, Crespo-Avilan GE, et al. Inflammation following acute myocardial infarction: multiple players, dynamic roles, and novel therapeutic opportunities. Pharmacol Ther. 2018;186:73-87. doi:10.1016/j. pharmthera.2018.01.001

5. Huang S, Frangogiannis NG. Anti-inflammatory therapies in myocardial infarction: failures, hopes and challenges. $\mathrm{Br} J$ Pharmacol. 2018;175(9):1377-1400. doi:10.1111/bph.14155

6. Ridker PM, Everett BM, Thuren T, et al. Antiinflammatory therapy with canakinumab for atherosclerotic disease. $N$ Engl $J$ Med. 2017;377(12):1119-1131. doi:10.1056/NEJMoa1707914

7. Mouton AJ, Rivera OJ, Lindsey ML. Myocardial infarction remodeling that progresses to heart failure: a signaling misunderstanding. $\mathrm{Am}$ J Physiol Heart Circ Physiol. 2018;315(1):H71-H79. doi:10.1152/ ajpheart.00131.2018

8. Frangogiannis NG. Cell biological mechanisms in regulation of the post-infarction inflammatory response. Curr Opin Physiol. 2018;1:7-13. doi:10.1016/j.cophys.2017.09.001

9. Zhou Y, Do DC, Ishmael FT, et al. Mannose receptor modulates macrophage polarization and allergic inflammation through miR-511-3p. J Allergy Clin Immunol. 2018;141(1):350-364. doi:10.1016/j.jaci.2017.04.049

10. Van der Borght K, Lambrecht BN. Heart macrophages and dendritic cells in sickness and in health: a tale of a complicated marriage. Cell Immunol. 2018;330:105-113. doi:10.1016/j.cellimm.2018.03.011

11. Li J, Song Y, Jin JY, et al. CD226 deletion improves post-infarction healing via modulating macrophage polarization in mice. Theranostics. 2020;10(5):2422-2435. doi:10.7150/thno.37106

12. Zhang N, Cui Y, Li Y, Mi Y. A novel role of nogo proteins: regulating macrophages in inflammatory disease. Cell Mol Neurobiol. 2021. doi:10.1007/s10571-021-01124-0

13. Crawford JR, Trial J, Nambi V, Hoogeveen RC, Taffet GE, Entman ML. Plasma levels of endothelial microparticles bearing monomeric C-reactive protein are increased in peripheral artery disease. J Cardiovasc Transl Res. 2016;9(3):184-193. doi:10.1007/ s12265-016-9678-0

14. Potempa LA, Rajab IM, Olson ME, Hart PC. C-reactive protein and cancer: interpreting the differential bioactivities of its pentameric and monomeric, modified isoforms. Front Immunol. 2021;12:744129. doi:10.3389/fimmu.2021.744129

15. Melnikov I, Kozlov S, Saburova O, et al. CRP is transported by monocytes and monocyte-derived exosomes in the blood of patients with coronary artery disease. Biomedicines. 2020;8(10):435. doi:10.3390/biomedicines 8100435

16. Slevin M, Iemma RS, Zeinolabediny Y, et al. Acetylcholine inhibits monomeric $\mathrm{C}$-reactive protein induced inflammation, endothelial cell adhesion, and platelet aggregation; a potential therapeutic? Front Immunol. 2018;9:2124. doi:10.3389/fimmu.2018.02124

17. Thiele JR, Zeller J, Kiefer J, et al. A conformational change in C-reactive protein enhances leukocyte recruitment and reactive oxygen species generation in ischemia/reperfusion injury. Front Immunol. 2018;9:675. doi:10.3389/fimmu.2018.00675

18. Wang J, Tang B, Liu X, et al. Increased monomeric CRP levels in acute myocardial infarction: a possible new and specific biomarker for diagnosis and severity assessment of disease. Atherosclerosis. 2015;239(2):343-349. doi:10.1016/j.atherosclerosis.2015.01.024

19. Schwedler SB, Amann K, Wernicke K, et al. Native C-reactive protein increases whereas modified $\mathrm{C}$-reactive protein reduces atherosclerosis in apolipoprotein E-knockout mice. Circulation. 2005;112 (7):1016-1023. doi:10.1161/CIRCULATIONAHA.105.556530 
20. Popena I, Abols A, Saulite L, et al. Effect of colorectal cancer-derived extracellular vesicles on the immunophenotype and cytokine secretion profile of monocytes and macrophages. Cell Commun Signal. 2018;16(1):17. doi:10.1186/s12964-018-0229-y

21. Vilela P, de Oliveira JR, de Barros PP, Leao MV, de Oliveira LD, Jorge AO. In vitro effect of caffeic acid phenethyl ester on matrix metalloproteinases (MMP-1 and MMP-9) and their inhibitor (TIMP-1) in lipopolysaccharide-activated human monocytes. Arch Oral Biol. 2015;60(9):1196-1202. doi:10.1016/j.archoralbio.2015.04.009

22. Barbero G, Castro MV, Villanueva MB, et al. An autocrine Wnt5a loop promotes NF-kappaB pathway activation and cytokine/chemokine secretion in melanoma. Cells. 2019;8(9):1060. doi:10.3390/ cells8091060

23. Chen T, Xu PC, Gao S, Hu SY, Wei L, Yan TK. Monomeric $\mathrm{C}$-reactive protein promotes platelets to release mitochondrial DNA in anti-neutrophil cytoplasmic antibody-associated vasculitis. Mol Immunol. 2021;137:228-237. doi:10.1016/j.molimm.2021.07.007

24. Aurora AB, Porrello ER, Tan W, et al. Macrophages are required for neonatal heart regeneration. J Clin Invest. 2014;124(3):1382-1392. doi:10.1172/JCI72181

25. Liu YC, Yao FH, Chai YF, Dong N, Sheng ZY, Yao YM. Xuebijing injection promotes $\mathrm{M} 2$ polarization of macrophages and improves survival rate in septic mice. Evid Based Complement Alternat Med. 2015;2015:352642. doi:10.1155/2015/352642

26. Cao Q, Wang Y, Wang XM, et al. Renal F4/80+ CD11c+ mononuclear phagocytes display phenotypic and functional characteristics of macrophages in health and in Adriamycin nephropathy. J Am Soc Nephrol. 2015;26(2):349-363. doi:10.1681/ASN.2013121336

27. Montaigne D, Marechal X, Modine T, et al. Daytime variation of perioperative myocardial injury in cardiac surgery and its prevention by Rev-Erb $\alpha$ antagonism: a single-centre propensity-matched cohort study and a randomised study. Lancet. 2018;391(10115):59-69. doi:10.1016/S0140-6736(17)32132-3

28. Xiao M, Zhang J, Chen W, Chen W. M1-like tumor-associated macrophages activated by exosome-transferred THBS1 promote malignant migration in oral squamous cell carcinoma. $J$ Exp Clin Cancer Res. 2018;37(1):143. doi:10.1186/s13046-018-0815-2
29. Lafuse WP, Wozniak DJ, Rajaram MVS. Role of cardiac macrophages on cardiac inflammation, fibrosis and tissue repair. Cells. 2020;10(1):51. doi:10.3390/cells 10010051

30. Wang S, Cao M, Xu S, et al. Luteolin alters macrophage polarization to inhibit inflammation. Inflammation. 2020;43(1):95-108. doi:10.1007/s10753-019-01099-7

31. Jung M, Ma Y, Iyer RP, et al. IL-10 improves cardiac remodeling after myocardial infarction by stimulating M2 macrophage polarization and fibroblast activation. Basic Res Cardiol. 2017;112(3):33. doi:10.1007/s00395-017-0622-5

32. Badimon L, Pena E, Arderiu G, et al. C-reactive protein in atherothrombosis and angiogenesis. Front Immunol. 2018;9:430. doi:10.3389/fimmu.2018.00430

33. Frangogiannis NG, Dewald O, Xia Y, et al. Critical role of monocyte chemoattractant protein-1/CC chemokine ligand 2 in the pathogenesis of ischemic cardiomyopathy. Circulation. 2007;115(5):584-592. doi:10.1161/CIRCULATIONAHA.106.646091

34. Liu S, Chen J, Shi J, et al. M1-like macrophage-derived exosomes suppress angiogenesis and exacerbate cardiac dysfunction in a myocardial infarction microenvironment. Basic Res Cardiol. 2020;115(2):22. doi:10.1007/s00395-020-0781-7

35. Riera-Borrull M, Cuevas VD, Alonso B, et al. Palmitate conditions macrophages for enhanced responses toward inflammatory stimuli via JNK activation. J Immunol. 2017;199(11):3858-3869. doi:10.4049/jimmunol.1700845

36. Han MS, Jung DY, Morel C, et al. JNK expression by macrophages promotes obesity-induced insulin resistance and inflammation. Science. 2013;339(6116):218-222. doi:10.1126/science.1227568

37. Lee $\mathrm{CH}$, Lui DTW, Lam KSL. Adipocyte fatty acid-binding protein, cardiovascular diseases and mortality. Front Immunol. 2021;12:589206. doi:10.3389/fimmu.2021.589206

38. Pan H, Huang W, Wang Z, et al. The ACE2-Ang-(17)-Mas axis modulates M1/M2 macrophage polarization to relieve CLP-induced inflammation via TLR4-mediated NF-small ka, Cyrillicb and MAPK pathways. J Inflamm Res. 2021;14:2045-2060. doi:10.2147/JIR. S307801
Journal of Inflammation Research

\section{Publish your work in this journal}

The Journal of Inflammation Research is an international, peerreviewed open-access journal that welcomes laboratory and clinical findings on the molecular basis, cell biology and pharmacology of inflammation including original research, reviews, symposium reports, hypothesis formation and commentaries on: acute/chronic inflammation; mediators of inflammation; cellular processes; molecular

\section{Dovepress}

mechanisms; pharmacology and novel anti-inflammatory drugs; clinical conditions involving inflammation. The manuscript management system is completely online and includes a very quick and fair peerreview system. Visit http://www.dovepress.com/testimonials.php to read real quotes from published authors. 